\title{
Specimen records of Bombus (Alpinobombus) in the Oregon State Arthropod Collection as of December 2018.
}

Riley Duncan

Oregon State Arthropod Collection

duncanri@oregonstate.edu

cite this work as:

Duncan, R. 2019. Specimen records of Bombus (Alpinobombus) in the Oregon State Arthropod Collection as of December 2018. Catalog: Oregon State Arthropod Collection 3(1). p 4-6.

http:// dx.doi.org/10.5399/osu/cat osac.3.1.4560

\section{Introduction:}

The gold-belted bumblebee, Bombus (Alpinobombus) kirbiellus Curtis 1835, is a poorly known and seldom collected species found in northern latitudes as well as some high elevation sites (Hatfield et al. 2014). As it currently stands, Alpinobombus Skorikov 1914 includes 9 valid species, 6 of which are found in western North America (Table 1). Of these, only Bombus kirbiellus (often referred to as $B$. balteatus Dahlbom, 1832) is regularly found in the United States outside of Alaska, with populations known from the both the Rocky Mountains and central California (Koch et al. 2015).

Table 1.

North American species of Bombus (Alpinobombus)

\begin{tabular}{|l|l|}
\hline Species & Present in the OSAC \\
\hline B. kirbiellius Curtis 1835 & 32 \\
\hline B. neoboreus Sladen, 1919 & 4 \\
\hline B. polaris Curtis 1835 & 10 \\
\hline B. natvigi Richards 1931 & - \\
\hline B. hyperboreus Schönherr, 1809 & 22 \\
\hline B. kluanensis Williams, Canning \& Corey 2016 & - \\
\hline
\end{tabular}

This dataset is part of a larger effort to generate digital label data for the entire bumblebee collection at Oregon State University and is part of several ongoing collaborations related to pollinators and pollinator health in the Pacific Northwest, such as the Oregon Bee Project

(https://www.oregonbeeproject.org).

\section{Methods/Metadata}

Each specimen is given a unique serial number (catalog number) that is printed as a machinereadable barcode, as well as a human readable numeral, on an acid-free, card-stock label that is placed as the bottom-most label. 
Data standards: All label data is transcribed into fields that are consistent with Darwincore standards for occurrence data (http://rs.tdwg.org/dwc/terms/Occurrence).

Taxonomic treatment: the taxonomic identity for all specimens is based on the historical determination labels associated with those specimens. Specimens were not re-determined as part of this project. Metadata about the determination is provided using Darwincore Identification fields (http://rs.tdwg.org/dwc/terms/Identification). Many specimens do not bear individual determination labels but are present in labeled unit trays. In these cases, the Identifiedby field is left blank although the collection was originally curated by William P. Stephen who studied bumblebees for many years at Oregon State University. Nomenclature follows that of Williams et al. (2015).

Locality and georeference data. Locality data was transcribed into the DublinCore location fields (http:/ / purl.org/dc/terms/Location); namely: country, stateProvince, county and locality. Missing information (e.g., no county printed on actual specimen label) was added to a record only if it could be determined without ambiguity based on gazetteers, maps or in reference to other label data. Elevational data on labels were converted to minimumElevationInMeters. Elevational ranges (e.g, $500-1200 \mathrm{~m}$ ) were recorded as the lower of the two. Georeferencing for locality data was conducted by searching GoogleEarth for the most appropriate placenames and recording the decimal degree latitude and longitude associated with that point. Because of the ambiguity in this approach it is recorded at a tenth of a degree

Metadata: Record type is considered physicalObject and basisofRecord is entered as preservedSpecimen. The collector(s) of the specimen are entered in the recordedBy field. The OccurrenceID is a URI for the specimen record itself, and includes the catalog number: "http:/ / osac.oregonstate.edu/SP/OSAC_XXXXXXXXXX" where "XXXXXXXXXX" is the 10-digit unique catalog number for the specimen.

\section{Results}

One hundred and eight specimens of Alpinobombus were found in the holdings of the OSAC, belonging to 6 species, with 4 of the 6 North American species being represented (Table 1). Nineteen specimens were not identified to species level. Label data for 20 specimens were not included in the supplemental dataset, as their labels lacked appropriate locality information or could not be interpreted reliably at this time.

\section{Discussion}

These data are presented for use by researchers and conservationists interested in Bombus (Alpinobombus) historical records. They are available for use either via download from the supplemental file associated with this publication (.csv text format) or will be shared online via an IPT server, accessible through GBIF (www.gbif.org). Access via GBIF is recommended, as that dataset will include the most recent version of these records in the case that they are modified/updated at a later date. Use of any of these records, in print or in online datasets and websites, should cite the dataset appropriately. Comments, edits or corrections to particular data records should be sent to osac@science.oregonstate.edu or the current curatorial staff of the Oregon State Arthropod Collection so they may be updated. 


\section{References}

Hatfield R, Colla S, Jepsen S, Richardson L, Thorp R, Foltz Jordan S (2014) IUCN Assessments for North American Bombus spp., pp 1-56.

https:// www.xerces.org/wp-content/uploads/2014/12/North-American-BombusRed-List-assessments-10-2014.pdf

Koch J, Lozier J, Strange J, Ikerd H, Griswold T, Cordes N, Solter L, Stewart I, Cameron S (2015) USBombus, a database of contemporary survey data for North American Bumble Bees (Hymenoptera, Apidae, Bombus) distributed in the United States. Biodiversity Data Journal 3. doi: https:// doi.org/10.3897/BDJ.3.e6833

Williams PH, Byvaltsev AM, Cederberg B, Berezin MV, Ødegaard F, Rasmussen C, Richardson LL, Huang J, Sheffield CS, Williams ST (2015) Genes suggest ancestral colour polymorphisms are shared across morphologically cryptic species in arctic bumblebees. Plos one 10: e0144544. doi:2015https:// doi.org/10.1371/journal.pone.0144544

\section{Acknowledgements}

Supported by the Foundation for Food and Agriculture Research under award number: 549024. The content of this publication is solely the responsibility of the authors and does not necessarily represent the official views of the Foundation for Food and Agriculture Research. 